\title{
Strategic behaviour in Schelling dynamics: Theory and experimental evidence
}

\author{
Juan M. Benito-Ostolaza ${ }^{\mathrm{a}}$, Pablo Brañas-Garza ${ }^{\mathrm{b}, *}$, Penélope Hernández ${ }^{\mathrm{c}}$, Juan A. \\ Sanchis-Llopis ${ }^{\mathrm{d}}$ \\ ${ }^{a}$ Departamento de Economía. Universidad Pública de Navarra, Campus Arrosadia s/n. 31006 \\ Pamplona. Navarra. Spain. \\ ${ }^{b}$ Middlesex University London, Business School, London NW4 4BT, England. \\ ${ }^{c}$ Departamento de Análisis Económico y ERI-CES, Facultad de Economía. Avda. dels Tarongers, \\ s/n.46022 Valencia. Spain.e-mail: penelope.hernandez@uv.es. \\ ${ }^{d}$ Departamento de Estructura Económica y ERI-CES, Facultad de Economía. Avda. dels \\ Tarongers, s/n. 46022 Valencia. Spain.
}

\begin{abstract}
In this paper we experimentally test Schelling's (1971) segregation model and obtain the striking result of full segregation in most of the cases. In addition, we extend Schelling's model theoretically by adding strategic behaviour and moving costs. We obtain a unique subgame perfect equilibrium in which rational agents facing moving costs may find it optimal not to move (anticipating other participants' movements). This equilibrium is far from full segregation. We run experiments for this extended Schelling model, and find that the percentage of full segregated societies is notably reduced when the cost of moving is high, but it is not affected when it is low, relative to the baseline with costless moving. We also find that the degree of segregation depends on the distribution of strategic subjects relative of a baseline model where moving is costless.
\end{abstract}

Keywords: Subgame perfect equilibrium and segregation and experimental games.

JEL Codes: C72, C9, R23.

\footnotetext{
${ }^{*}$ Corresponding author

Email address: branasgarza@gmail.com (Pablo Brañas-Garza)
} 


\section{Introduction}

Segregation is a relevant economic, political and social problem all around the world, as it enhances marginalization, poverty, illiteracy, etc. Examples such as favelas in Brasil or red districts at Pattaya and Chonburi (Thailand) do not need further explanations. ${ }^{1}$

Individuals with similar ideas, habits, preferences, political affiliations or from the same ethnic group tend to join together and create cliques and clusters in communities, which result in segregation at the society scale. Thomas C. Schelling (1971) provided the first model of spatial segregation. He showed that even individuals with very low mixing aversion may cause a segregated society in dynamic environments. ${ }^{2}$

Segregation can be the result of individual preferences but also the maximizing social welfare configuration. In other words, an equilibrium configuration can exhibit high levels of segregation, although there are not individuals' preferences for segregation per se. This phenomenon illustrates unintended consequences resulting from the interaction between individuals.

The basic prediction of Schelling's model is that under some general conditions it is nearly impossible to fight against a high level of segregation ${ }^{3}$. With individuals acting in their own self interest we have a socially suboptimal configuration.

This paper provides a new and more optimistic result. When subjects are completely rational and moving is costly then the full segregated configuration is not an equilibrium.

\footnotetext{
${ }^{1}$ Programs such us "Moving to the Opportunity" indicate how strong is the social concern about segregation in the U.S. (for more references see Kling, Liebman and Katz, 2007).

${ }^{2}$ Schelling defined a model in which agents, divided into two types, move on a checkerboard according to a given utility function. Within this set up, Schelling shows that segregation occurs even when individuals have very mild preferences for neighbours of their type, as long as they are allowed to move in order to satisfy their preferences.

${ }^{3}$ The Schelling segregation benchmark consists of a spatial model in which agents of two welldifferentiated types distribute along a line with preferences that depend on the composition of their surrounding neighbourhoods. In this model there are no objective neighbourhood boundaries; individuals define their neighbourhood with respect to their location. An individual moves if she is not content with the type mixture of her neighbourhood, moving to a place where the mixture of individuals meets her tolerance level, which is defined as the proportion of individuals of different types in her neighbourhood. Schelling's seminal model strikingly predicts a high segregation outcome from the initial situation when agents are myopic and can move costlessly.
} 
How can we get this result? Individuals in the Schelling model are myopic. We consider as myopic agents those individuals who move according to the Schelling specific rule: individuals move whenever they are not content. So, they do not compute too much and they just respond instinctively (see Rubinstein, 2007). However, in our model we check whether the dynamics of the model change when we do not assume such non-elaborated reasoning. Surprisingly, when we model the Schelling dynamics using strategic -instead of myopic players- we obtain an unexpected result: we do find that less segregated outcomes, than the ones predicted by Schelling, are also equilibria. Hence, under rationality, the full segregated society is not the unique equilibrium.

Theoretically it would be highly desirable to find an environment in which society members' incentives are aligned around a unique equilibrium. This problem is already solved when we introduce costly moving. ${ }^{4}$ We find that the introduction of any positive cost in the strategic model solves the multiple equilibria problem. This equilibrium with moving costs constitutes our first result.

The introduction of moving costs makes the model closer to reality, where the costs of moving play a crucial role on individuals' decisions, ${ }^{5}$ and solves the theoretical problem aforementioned.

The second contribution of this paper is experimental. We design an experiment to unravel how individuals play when moving is costly. We use a one-shot game where 8 subjects are randomly placed (face-to-face) around a real circle describing an unhappy society configuration: black, white, black, white, etc. We call a configuration in which at least one individual may improve her happiness by changing her location an unhappy society. In our setting subjects are given the chance to move or stay in order to reach the maximum level of happiness in the form of a fixed monetary payoff.

With the spirit of capturing the real decision of moving we introduce low and high moving costs. We compare this with the baseline model of no costs.

Our experimental data show that the Schelling outcome does not always emerge,

\footnotetext{
${ }^{4}$ The assumption of free moving in the original model is restricted to minimal movement (the nearest place). Therefore, Schelling is assuming that, in certain sense, moving is not completely costless.

${ }^{5}$ Individuals are utility maximizers, and they may be indifferent to several actions if they get the same payoff. However, the existence of costs that may reduce their benefits may make them to behave more strategically. Therefore, strategic agents facing moving costs may anticipate subsequent movements by other participants, finding it optimal not to move.
} 
especially if there are moving costs. The existence of moving costs fosters the appearance of individuals behaving strategically. Therefore, we also show that strategic behaviour is not rare but, on the contrary, quite abundant in treatments with moving costs. And most importantly, the fully segregated outcome vanishes in the presence of strategic players.

The rest of the paper is organized as follows. The related literature is presented in section 2. Section 3 is divided into three subsections. In the first subsection we recall the classic Schelling linear model, in the second subsection we present our extensive Schelling dynamic game and, finally, we introduce two definitions of individual behaviour in the third subsection. The experimental design is explained in section 4 , results are presented in section 5 , and section 6 concludes.

\section{Related Literature}

Using one and two-dimensional landscapes, Schelling $(1969,1971)$ showed the emergence of high segregation even if individuals in the society had mild preferences for living with neighbours of their own type. Schelling's result is, in general, of interest to economists, policy makers and social scientists because it illustrates the emergence of an aggregated phenomenon that cannot be directly foreseen from individual behaviour and concerns an important problem: segregation. This striking result has generated a vast amount of literature from a wide range of scientific trends. Miltaich and Winter (2002), assuming that individual's characteristics are unidimensional, find a stable partition that not only is stable but also segregating. Likewise, Karni and Schneidler (1990) examine the conditions for segregation and group formation in an overlapping generations model. On the other hand, the seminal concept of stochastic stability introduced by Foster and Young (1990), and developed within the evolutionary game theory literature, provides generations insight into Schelling's spatial proximity model. Young $(1998,2001)$ presents a simple variation of the one-dimensional Schelling model, showing that segregation tends to emerge in the long run, even though a segregated neighbourhood is not preferred by any agent. Zhang (2004) extends Young's set-up (1998) into a two-dimensional framework. Both studies argue that complete segregation is the only viable long-run outcome for best-response dynamics if the agents' preferences are biased in favor of their own type. Pancs and Vriend (2007) also find that complete segregation is the only possible long-run outcome in a ring where agents have balanced preferences about the racial composition of their neighbourhood. Although the analytical result in Pancs and Vriend (2007) cannot be extended to a two-dimensional society setting, 
they show, through simulations, that best-response dynamics also tend to produce segregation even in a two-dimensional space.

In summary, this branch of the literature shows that even if all individual agents have a strict preference for perfect integration, myopic best-response dynamics may lead to segregation ${ }^{6}$. This finding casts some doubts on the design (ability) of public policies to improve integration by promoting openness and tolerance with respect to diversity.

On the empirical side, many studies on racial segregation analyze discrimination in housing prices. Specifically, studies from the 1960s, such as King and Mieszkowski (1973), tend to find evidence that African-Americans pay more for equivalent housing. However, studies from the 1970s, such as Follain and Malpezzie (1981), do not confirm this evidence. Cutler, Glaeser and Vigdor (1999) confirm that the AfricanAmerican rent premium fell dramatically between 1940 and 1970 and had reversed entirely by 1990 .

Another branch of the empirical literature explains segregation through social interaction models. In this literature, the concept of tipping ${ }^{7}$ is crucial for understanding the dynamics of segregation. In particular, segregation emerges and persists precisely because such residential patterns resist tipping. Clark and Fossett (2007) provide simulation experimental results crafted to explore the implications of ethnic preferences in multi-group situations. They establish that ignoring the role of choice behaviour based on own-race preferences is akin to omitting the potentially important influence of racial and ethnic dynamics in residential composition. Using regression discontinuity methods and Census tract data from 1970 through 2000, Card, Mas and Rothstein (2008) find strong evidence that white population flows exhibit tipping-like behaviour in most cities of the U.S. This result is consistent with that of Cutler, Glaeser and Vidgor (1999) and Card, Mas and Rothestein (2008), who find that tipping points are significantly higher in cities with higher minority shares.

In sum, the empirical evidence also points to the existence of high segregation even when agent preferences depend on individual choices and every agent prefers to live in a mixed-race neighbourhood.

\footnotetext{
${ }^{6}$ In the above literature, the main assumption about individual behaviour is that individuals do not behave rationally.

${ }^{7}$ Tipping is said to occur when some recognizable minority group in a neighbourhood reaches a size that motivates other residents to leave.
} 


\section{Theory}

Schelling's $(1969,1971)$ linear model considers a finite number of individuals distributed along a line where the individuals are of black or white types. ${ }^{8}$ All members of the population are assumed to care about the individuals' typology they live with, i.e., their neighbourhood. Everyone is able to move to another location if she is dissatisfied with the type mixture she lives in. Specifically, each agent defines her neighbourhood as the $d>0$ individuals on either side of her own location. Therefore each agent's neighbourhood is composed of her $2 d$ adjacent neighbours. Schelling assumed that every agent prefers to have at least $m \in\{1, \ldots, 2 d\}$ neighbours of her same type. We call $m$ the individual's tolerance level ${ }^{9}$, which represents a threshold over the composition of each agent's neighbourhood. Therefore agents' preferences over their neighbourhoods are defined over the parameters $d$ and $m$. Through these parameters we can determine whether an individual is happy (if the number of neighbours like her is larger than or equal to $m$ ) or unhappy (if the number of neighbours like her is smaller than $m) .{ }^{10}$

Unhappy agents move in turns, should they still be unhappy when their turn comes, starting from the left to the nearest place that fulfills their neighbourhood configuration demand. Schelling defined nearest place as the point reached by surpassing the smallest number of neighbours on the way. In those cases in which an agent has two nearest places at the same distance (one in each side), the choice is arbitrary. In this setting it is assumed that moving is costless.

Whenever one agent moves, two different situations may arise. First, someone who was happy may become unhappy because like members move out from her neighbourhood (or opposite members move in). Second, those who were initially unhappy are now happy as opposite neighbours move away (or like neighbours move nearby). Moreover, the Schelling rule holds that, in each round, any initially unhappy member who is happy when her turn comes will not move. Likewise, anyone who becomes discontent in the previous round will have her turn after all initially discontents have had their innings. This process stops when no agent wants to move anymore or continue ad infinitum.

\footnotetext{
${ }^{8}$ In the one-dimensional model, Schelling (1971) also refers to the possibility of considering an infinitely continuing line or a ring.

${ }^{9}$ Young (2009) referred to this concept as the agent $i$ 's social threshold.

${ }^{10}$ The parameters $d$ and $m$ allow to examine different examples as set out in Schelling (1971), where different neighbourhood sizes may have some different requirements (for example, if we have a neighbourhood composed by four neighbours we want at least one like us, if it is composed by six we want at least two, ...).
} 
Note that, in the Schelling setting nobody anticipates the movements of others. That is, when their turn to move comes, individuals move if their neighbourhood demands are not met, ${ }^{11}$ and the typical outcome is a highly segregated society. The dynamics in Schelling's model can be defined as an iterative and sequential process of agents choosing myopic responses, where the only restriction to the mobility of agents is to go to the nearest place.

\subsection{Schelling's linear model}

Let us consider a very simple case to understand the apparently simple dynamics of Schelling's linear model. Suppose that a society is composed of 8 individuals of two types, of which four are blacks (B) and four are whites (W). Assume that these individuals are distributed along a ring -that is, a society- with the following configuration:

$$
\{B, W, B, W, B, W, B, W\}
$$

Let us denote the individuals as their location on (1) starting from left to right. Hence, agent 1 is the top black bullet, agent 2 is the next white bullet and so on until agent 8 , who will be the last white bullet. This configuration is represented as a connected ring in Figure 1.

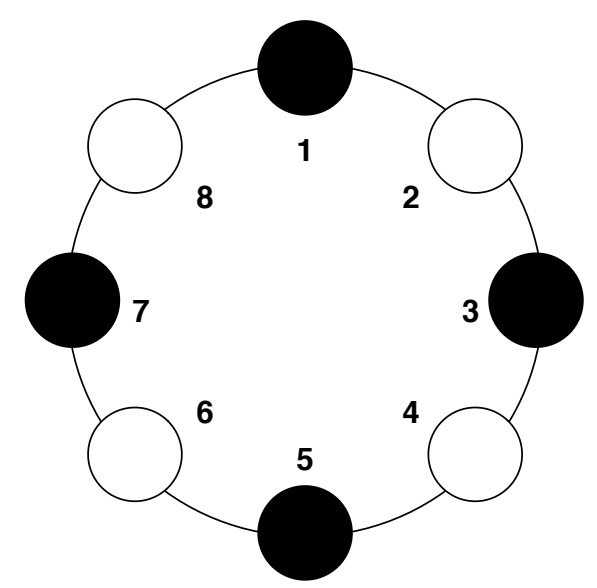

Figure 1: A particular initial configuration of four type B individuals and four type W individuals.

Furthermore, let's suppose that each individual in configuration (1) accepts up to

\footnotetext{
${ }^{11}$ This coincides with our definition of a myopic player.
} 
$50 \%$ of unlike neighbours ${ }^{12}$ over a neighbourhood composed of one individual at each side $(d=1)$. Notice that no player is happy in the initial configuration of the society and therefore everybody is willing to move. Let's see now how Schelling's dynamics work.

The first unhappy individual is agent 1, who is not satisfied with her neighbourhood configuration. She may move to two satisfactory positions: either the position between agents 2 and 3 or the position between agents 7 and 8 . As stated before, we solve this symmetric case by moving agent 1 to the right. That is, agent 1 moves between agents 2 and 3 (Figure 2(a) below). After makes this move agent 1, agents 2, 3 and 8 become happy. Therefore the next unhappy agent in the ring is individual 4. This agent will move to the location between 5 and 6 (Figure 2(b)), thereby making agents 5 and 6 happy. The next unhappy agent, and in this case the last one, is agent 7 . Agent 7 will move to the position between agents 2 and 1 , as it is the nearest position to the right that fulfills her preferences (Figure 2(c)). Notice that although individuals $2,3,5,6$, and 8 were initially unhappy, they do not move because they were happy when their turns to move came. This process ends at this stage because all individuals are happy, and no one wants to move to another location (Figure 2(d)). Thus, the society ends up in a situation of full segregation.

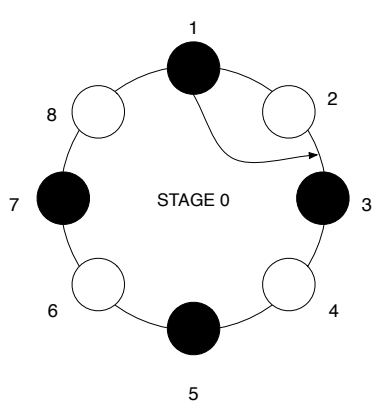

(a)

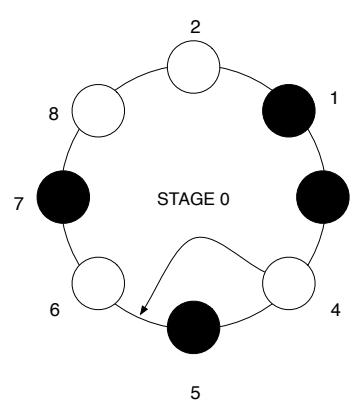

(b)

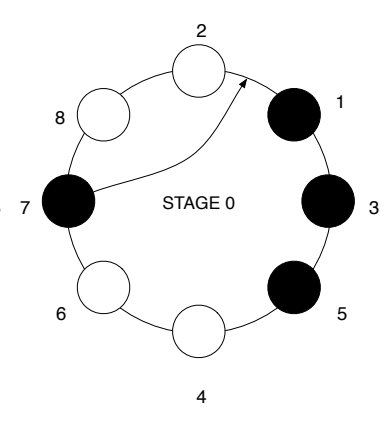

(c)

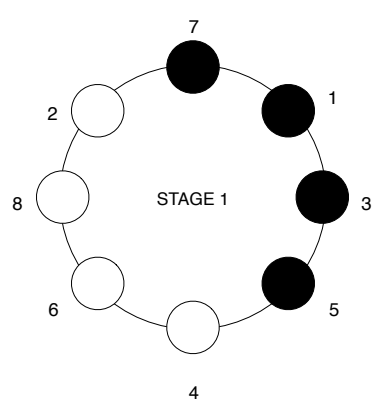

(d)

Figure 2: Figures (a), (b), (c) and (d) illustrate the dynamics of Schelling's myopic response over the particular case of Figure 1.

\footnotetext{
${ }^{12}$ As in the original Schelling model. Schelling $(1969,1971)$ also considers the possibility that agents accept up to other percentages of unlike neighbours.
} 


\subsection{Schelling model with fully rational players}

Now, we study the impact of strategic behaviour in the Schelling's linear model under two scenarios: without and with moving costs. Let's start with the model without moving costs.

Each player $i \in\{1,2, \ldots, N\}$ has two possible actions: either "to stay" at her initial location denoted by $\underline{i}$ or "to move" to the nearest space with a neighbour of her same type denoted by $\bar{i}$. Each player prefers to be close to at least one like neighbour. Given these preferences, we translate the agent's preferences into a utility function that depends on, the individual's actions and the final configuration of the society. In particular, the payoff for every player is defined as a positive value, $M$, if the agent ends up with at least one neighbour like her. Recall that in the Schelling's model there exists a moving order for each unhappy player. The first unhappy player moves, then the second agent faces the same decision and so on until the last unhappy agent. The dynamic structure described above could be modeled as an extensive game where the players play sequentially. In such a setting, each agent takes her best-response action. That is, one player could find it optimal either "to stay", because she anticipates that the actions of the other players would generate a final configuration where she will become happy, or "to move", otherwise. Both actions are allowed in our model, and an unhappy player does not necessarily have to move. Moreover, given the payoff structure of the game the behaviour of player $i$ will explicitly depend on both $d$ and $m$.

This explicit scenario, seen as a sequential game, is denoted by $\Gamma_{0}^{n}$. The proper equilibrium notion for the above dynamic scenario is the subgame perfect equilibrium (SPE henceforth). The equilibrium strategies should specify optimal behaviour from any information node up to the end of the game. That is, any agent's strategy should assign what is optimal from that node onwards, given her opponents' strategies.

When there are no moving costs, any final configuration where any individual is happy may be implemented by different sequences of actions and all of then are equilibria. For instance, for $d=m=1$, the sequence of actions explained in the above subsection (where player 1,4 and 7 move and the remainder players stay) is an equilibrium path. But there are more possibilities, for instance a situation where player 2 moves between 4 and 5 and player 6 moves between 7 and 8 is as well an equilibrium. In this case, the final configuration $B, B, W, W, B, B, W, W$ is a happy society since every player ends with at least a neighbour like her.

The following lemma asserts the existence of pure equilibria in $\Gamma_{0}^{n}$ for all $d, m$. 
Notice that when $n$ is small and there are not enough agents of one type to satisfy the happiness condition, then there is not a happy final configuration and to stay is the best strategy for these agents. Notice that the game $\Gamma_{0}^{n}$ is a finite extensive game under complete information, hence there are pure equilibria ${ }^{13}$. The formal presentation of the game is in Appendix A.

Lemma 1. Let $m, d, n \in \mathbb{N}$ such that $0 \leq m \leq 2 d$ and $n \geq 2(m+1)$. The game $\Gamma_{0}^{n}$ with players of each type (at least $m$ ) has equilibria in pure strategies.

Next subsection illustrates how positive costs have an impact on the set of equilibria. Actually, a strong refinement is achieved for $d=m=1$ for a particular initial society.

\subsection{Fully rational players and moving costs}

We extend the game by introducing moving costs, $C>0$, to modify the value of the payoff function. Thus, if at the very end of the game player $i$ is happy, she earns the payoff $M$ if she has moved or the payoff $M+C$ if she has not moved. If she ends up in an unhappy situation, she gets 0 or $C$ if her action was "to move" or "to stay", respectively. We assume that $M>C>0$.

As the setting without moving costs, we can see this explicit scenario as a sequential game that we denote by $\Gamma^{n}$. We can study the SPE and the corresponding equilibrium strategies which will depend on both $d$ and $m$.

Consider first the following case: let $\{\mathrm{B}, \mathrm{W}, \mathrm{B}, \mathrm{W}, \mathrm{B}, \mathrm{W}, \mathrm{B}, \mathrm{W}\}$ be an initial configuration. If $d=m=2$ such a configuration will be a happy configuration since all their individuals have two neighbours (with distance equal to 2) like her. Nevertheless, if $d=m=1$ then the same configuration is totally unhappy since any individual has only one neighbour like her. Therefore, the same configuration can be considered either happy or unhappy depending on the specific tolerance level.

Schelling establishes a behaviour when a player is happy and when she is not. Namely, if an individual is unhappy she will move to the nearest place where she becomes happy. Nevertheless, if she is happy she will stay at the same location. Actually, the movement of distance zero is the nearest place where she is (already) happy. Under strategic consideration we may find all the possible responses, conditional on the tolerance level. Let us see some examples to understand the scope of

\footnotetext{
${ }^{13}$ This lemma does not provide a characterization of the final configuration attending any initial configuration and conditions on $d, m$ since this is a well recognized complex problem.
} 
strategic individual behaviour.

Consider a history of length 6 generating the following configuration $\{W, W, B, W, B, W, B, B\}$ from the initial configuration $\{W, W, B, B, W, W, B, B\}$ with $d=m=2$. The game is at one node where player 7 with type $B$ is called upon to play. She has one neighbour like her with distance two to the left and another one with distance one to the right. Therefore, she is already happy. Schelling would declare "to stay" as the action played by player 7 . But would player 7 play "to stay" if player 8 were rational?

Suppose that player 7 follows the behaviour predicted by Schelling, i.e., she does not move. Then, the best response for player 8 is "to move" since player 8 is at the last stage of the game and she is still unhappy. The final configuration would be $\{W, W, B, B, W, B, W, B\}$ being the last one player 7 . It is easy to compute that player 7 would finish being unhappy. Nevertheless, if player 7 anticipates the best choice that player 8 will choose, then player 7 could see that by moving between player 1 and 2 the next configuration would be $\{W, B, W, B, W, B, W, B\}$. In this configuration player 8 is the last $B$ and player 7 is at position 2. Both players have two neighbours like them at distance two. In the last stage player 8 has no incentive to move and this would be the end of the game. ${ }^{14}$

Suppose now that $d=m=1$ and the initial configuration is $\{B, W, B, W, B, W, B, W\}$.

- If a player $i$ is already happy when she is called upon to play, then happiness only comes from the previous movements of players $1,2, \ldots i-1)$. Notice that the next neighbour $i+1$ has a different type than $i$. In this case, the best response is always "to stay".

- If $i$ is unhappy when she is called upon to play, then she has to compute her best response in the corresponding subgame. She has to check whether to stay or to move is her best response.

For instance, suppose that $h_{5}=(\underline{1}, \underline{2}, \underline{3}, \overline{4}, \underline{5})$ is the history for player 6 at node $s$. As player 6 is already happy when she is called upon to play, since player 4 has moved close to her, then, her best response is "to stay", $\underline{6}$, and her payoff would be $M+C$, given that she did not pay any moving cost.

\footnotetext{
${ }^{14}$ This case illustrates that within our framework, strategic behaviour and moving costs, a happy player may have incentives to move should she anticipate an agent playing after her will move leaving her in an unhappy position. Therefore, a positive cost does not necessarily imply a reduction of movements.
} 
Suppose now that player 6 is at node $s$ after the new history $h_{5}^{\prime}=(\underline{1}, \underline{2}, \overline{3}, \underline{4}, \underline{5})$. In this case, player 6 is unhappy. Is "to stay" the best response for her? As the action to move results in a lower payoff for any final situation, let's calculate if $\underline{6}$ is the best response for player 6 .

According to the definition of subgame perfection, it is necessary to find the best response for players 7 and 8 at the corresponding subgame denoted by $\Gamma_{h_{5}}$. Let's start by studying the best response for player 8 :

- Suppose that player 7 moves. Player 8 is then happy since player 6 is in her neighbourhood. Therefore the best response for player 8 is "to stay".

- Suppose that player 7 does not move. Then the best response for player 8 given the history $(\underline{1}, \underline{2}, \underline{3}, \overline{4}, \underline{5}, \underline{6}, \underline{7})$ is "to move" since player 8 is at the last stage of the game and she is still unhappy.

Now consider the best response of player 7 given the above best response for player 8.

- If player 7 is happy, then her best response is obviously "to stay".

- If player 7 is unhappy, taking into account the best strategy for player 8 , the corresponding best response will be "to stay" since it guarantees her the maximum payoff $(M+C)$.

Consequently, as player 7 will not move if agent 6 plays "to stay", she will end up unhappy. Hence, her best response is "to move", thus guaranteeing a happy position.

The above argument can be carried out at any (information) node for each player generating the SPE. In the previous examples, any configuration with any tolerance level generates an extensive game with the common property of no indifference condition at any information node. ${ }^{15}$ Moreover, if there are enough players to guarantee the happiness condition for any type then the final configuration is a happy society. This entails that the full rationality assumption supports a one-round extensive game. Namely, players only need to play once to reach happiness in contrast to the Schelling dynamics that may be infinite.

The following theorem states the existence of a unique SPE for $\Gamma^{n}$ for any tolerance level. Moreover, the case of $d=m=1$ for the initial configuration $(B, W, B, W, B, W$, $B, W)$ is studied. This instance is the simplest one that collects the main features

\footnotetext{
${ }^{15}$ Note that the game $\Gamma$ is a generic game.
} 
to properly discriminate between strategic versus myopic behaviour. Furthermore, the equilibrium path that arises from the unique SPE does not generate a completely segregated configuration in contrast to the fully segregated outcome reached in Schelling's model.

Theorem 1. - There exists a unique subgame perfect equilibrium in the extensive game $\Gamma^{n}$.

- For $d=m=1$ and the initial configuration $\{B, W, B, W, B, W, B, W\}$, the final configuration $\{B, B, W, W, B, B, W, W\}$ is the consequence of the unique equilibrium path $(\underline{1}, \underline{2}, \underline{3}, \overline{4}, \underline{5}, \underline{6}, \underline{7}, \overline{8})$.

The proof is in the Appendix.

The configuration reached by strategic players playing the SPE for a initial configuration $\{B, W, B, W, B, W, B, W\}$ and $d=m=1$ has two remarkable properties. On the one hand, any player is happy at the end of the sequential game and, on the other hand, half of the players decide to stay, thus avoiding the moving cost. We call this final configuration $\{B, B, W, W, B, B, W, W\}$ a happy-non-segregated (HNS henceforth) society. We should point out that this is the only happy configuration when there are moving costs.

\subsection{Summary}

Schelling provided an early contribution, proposing a model to formalize the aggregate consequences of individual preferences related to the social environment (Schelling, 1969, 1971). The basic components of Schelling's Dynamic models of segregation (1971) are an individual utility function that entirely determines the level of satisfaction enjoyed by an agent in a location, and a dynamic rule that drives agents' location changes and, therefore, the way the configuration of the city evolves. All this without cost of movement. Using an inductive approach, Schelling shows that if the preferences considered are such that an environment of more than $50 \%$ of like-group agents is highly preferred to an environment of less than $50 \%$, then the equilibrium configuration exhibits high levels of segregation, although there is no preference for segregation per se. Schelling's (1971) paper is a seminal work in relation to his seemingly paradoxical finding that mild individual preferences for own-group neighbours lead to complete segregation at the global level.

However, on reflection, we realize that, given equal payments between being myopic and not (as in Schelling's model, 1971), the final configuration of the society would hardly be an integrated environment. The introduction of the costs of moving in our setting discriminates between myopic individuals and those who are not, making 
it possible to achieve more integrated societies. Since real societies are comprised by heterogeneous individuals, our model can be seen as an extension of Schelling's model, illustrating how heterogeneous individuals can reach both segregated environments and not segregated ones.

Our theoretical contribution is focused on this specific case and it is not necessarily generalizable to any other environment. However, as we will see below, this theoretical set up provides the appropriate framework to explore subject's decisions.

\section{Experimental evidence}

This section describes the design of the experiments carried out to test: first, the existence of fully rational strategic players; and, second, how their behaviour affects the Schelling segregation result.

It is important to stress that the aim of this section is not proving that subjects play the SPE. We do not expect subjects will play in such a complex way. The aim of our experimental research is to check whether subjects use simple (myopic) or sophisticated (strategic) decision rules, and if the use of those rules by agents affects the final distribution achieved relative to the costless setting.

This section is divided in four subsections. First, we present the experimental design and some details about the implementation and the replication in the lab. Second, we properly define what is strategic and myopic behaviour in the specific game presented in the theoretical section. The third subsection is devoted to explain the results. A final subsection discusses our results.

\subsection{Experimental design}

We start with an experiment of the linear Schelling model without moving costs (baseline) and then provide an experiment of our extensive game with positive -low and high- moving costs (see section 3.3). The game played here shares some features with Benito et al. (2011), that analyzes the role of sequential and simultaneous moving in a Schelling experimental game without moving costs for 8 subjects. That paper compares the number of steps a society requires to reach the Nash equilibria. Although the theoretical prediction is that sequential movement requires less iterations than simultaneous movement (since information is higher in the later) the results are quite unexpected: In societies with simultaneous movement, we observe faster convergence or, in the worst case, we find no difference between sequential and 
simultaneous moving. Benito et al. (2011) extends the analysis to costly simultaneous moving, showing that the existence of positive costs make subjects less likely to move and, consequently, the convergence process is notably slowed down. It is crucial to note that Benito et al. (2011) does not provides any theoretical framework to analyze individual decision making.

\subsubsection{A face-to-face Schelling ring experiment}

Moving is a key ingredient of the Schelling model. Subjects choices are restricted either "to move" or "to stay". In our game, in an attempt to maintain the essence of individual choice, subjects physically move across a ring. We consider that a face-to-face experiment might better capture the features an individual takes into account when she decides to move. We are aware that anonymity is at risk in this environment. Therefore, to check whether there is any effect related to this issue we also run complementary computerized lab experiments (see section 4.1.4.).

We organize individuals in groups of eight around a circle, measuring 11.5 feet in diameter (see Figure 3). To allocate the individuals in each circle, we follow a random sorting scheme where the first individual is assigned the black type, the second individual the white type, and so on ${ }^{16}$, that is $\{B, W, B, W, B, W, B, W\}$. Each subjects' type is easily identifiable by a black/white scarf.

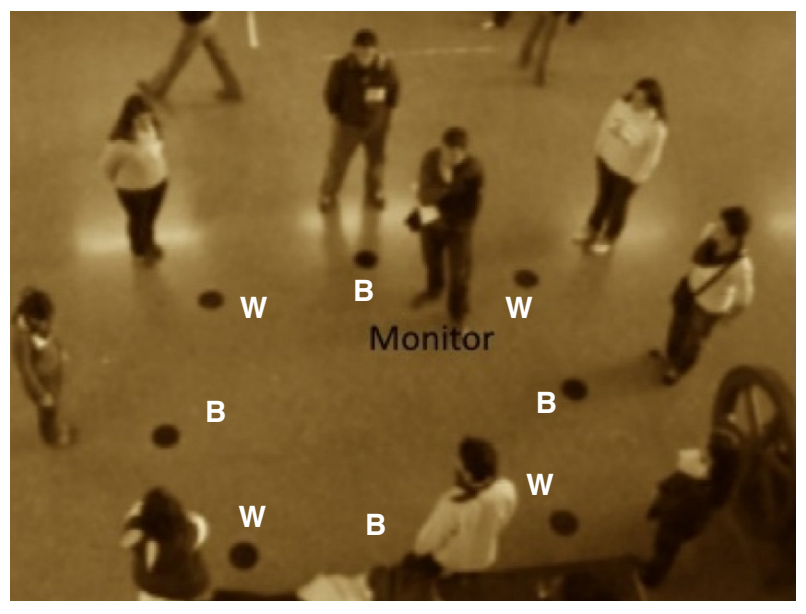

Figure 3: Circle configuration

\footnotetext{
${ }^{16}$ The experiment was conducted in Spain where the labels "black" and "white" are meaningless. Therefore there is no reason to be aware of potential framing.
} 
According to Schelling, players obtain positive utility if they reach a happy position (i.e., a player has at least one neighbour of her type). We capture the individual's utility function through a fixed prize that might be earned if a subject ends up with a like neighbour. Notice that in the initial configuration nobody is happy. Therefore, subjects have the opportunity "to stay" or "to move" from their location when their turn comes. Thus, subject 1 might decide to stay or to move $\{\underline{1}, \overline{1}\}$; after subject 1 has taken her decision, subject 2 (to the left of subject 1 ) faces an identical decision problem $\{\underline{2}, \overline{2}\}$; then, subject 3 , etc.

Individuals played the game five times. In each round, a random device places subjects in new positions (and therefore the color of their scarves changes accordingly). In the experiment, we use the following payoff scheme:

- players would be paid in one out of the 5 games (randomly selecting that game);

- two out of the eight subjects (we select that game randomly) would be paid; and,

- the prize would be 50 Euros for the happy subjects selected.

In summary, two out of the eight participants would be randomly selected to be paid 0 Euros if they end up being unhappy and 50 Euros otherwise.

\subsubsection{Face-to-face and costly Schelling treatments}

To implement the extensive Schelling dynamic game, we add moving costs to the above experiment. In particular, we run two treatments with moving costs:

T1: 5-Euros moving (low costs).

T2: 20-Euros moving (high costs).

We introduce the costs in the experiment in an easy way. We place the money on the floor in front of each subject and they are informed that, should they move they will lose this (potential) money. The analogy with real life costs is quite obvious: you leave your home or friends behind when you move. That is, all subjects can see a 5-Euros [20-Euros] bill at their feet. Consequently, if they move, they lose the 5 [20] Euros.

As before, two out of the eight participants are randomly selected to be paid. Therefore, in the extensive game: 
- An individual might earn 0 Euros if she ends up unhappy and has moved or 5 (20) Euros if she ends up unhappy and has not moved.

- If she ends up with a neighbour like her she might earn 50 Euros (if she has moved) or 50 plus 5 (20) Euros if she ends up with a neighbour like her and has not moved.

In addition to the 5 rounds (identical to the baseline with the only difference of the costs) we run another additional 5 rounds. ${ }^{17}$

\subsubsection{Implementation}

The experiment was ran at the School of Economics of the University of Granada (Spain) on April 20, 2009.

In particular, we ran 6 sessions or societies for each treatment (low and high costs) with 8 subjects in each society. Further, we run 5 sessions with 8 subjects for the baseline (no moving costs). Overall, we had a total of 136 participants ( 8 subjects $\times 6$ societies $+8 \times 6+8 \times 5)$. These participants were randomly recruited from a sample of 191 subjects who had signed up to participate in the experiment. ${ }^{18}$

All the experimental sessions were conducted the same day and at the same time. Experimental subjects were randomly assigned to each ring and they stayed in the same ring during the whole experiment. Seventeen associate professors, teaching assistants and Ph.D. students (working in experimental economics) acted as monitors to conduct the sessions. The monitors received identical training and had the same instructions booklet ${ }^{19}$ (including random assignments for each round, payoffs scheme, etc.).

As explained before, the baseline treatment, $c=0$, was repeated 5 times, therefore it provides information about $5 \times 5$ societies or $5 \times 5 \times 8$ individual data $(200$ observations). For treatments with costs, $c=5$ and $c=20$, we repeated the experiment after a surprise re-start, and therefore we have information about $(5 \times$ $6) \times 2$ societies or $(5 \times 6 \times 8) \times 2(480$ observations $)$ in both treatments.

\footnotetext{
${ }^{17}$ We repeated the game in order to increase the number of observations in our analysis. This repetition is done using a surprise restart. We do not change the rules or any other aspect of the game. Players have the chance to double their earnings as we pay one participant in one of the 5 rounds of the 2 sessions. Our results indicate that there is not any impact on the results (between the two sessions) and therefore we pool the data across the 2 sessions (10 rounds).

${ }^{18}$ In the following we will use the term society to refer to clocks.

${ }^{19} \mathrm{~A}$ copy of the instructions booklet is available upon request.
} 
Due to unintended errors by the monitors we missed some data: $i$ ) one of the monitors in $c=20$ did not run the repetition; $i$ i) two societies in $c=20$ were impossible to be read due to a large number of crossing out and erasures. As a result we lost some observations. Table 1 shows the number of experimental data per treatment (where the last two columns reflect the real data we use once we exclude the missing values).

The complete experiment lasted 1 hour and the average earning was 30 Euros. Before starting the real game, subjects played two trial runs to ensure that they understood the structure of the game.

Two important features stand out in our design:

1. The randomization sorting in each round allows us to obtain different types of actions for any given subject: i.e., first mover, second mover and so on.

2. This within-subject analysis enables us to explore how experience may alter subjects' willingness to play more strategically.

Table 1: Summary of treatments \& observations (face-to-face)

\begin{tabular}{|c|c|c|c|c|c|c|c|}
\hline & sessions & rounds & repetitions & societies & obs. & societies (real) & obs. (real) \\
\hline$c=0$ & 5 & 5 & 0 & 25 & 200 & 25 & 200 \\
$c=5$ & 6 & 5 & 1 & 60 & 480 & 60 & 480 \\
$c=20$ & 6 & 5 & 1 & 60 & 480 & 53 & 424 \\
\hline
\end{tabular}

Note: "obs." is the number of observations

\subsubsection{A non face-to-face Schelling ring experiment}

As economic incentives, anonymity is a crucial feature of experimental economics. Our face-to-face set-up clearly fails in this respect. To check the robustness of our results we ran a number of additional treatments with standard lab conditions. We ran a number of computerized sessions at the LINEEX experimental laboratory of the University of Valencia (Spain) on February, 2010. All of them played 10 rounds with identical instructions to the face-to-face experiment.

- 40 subjects participated in the baseline treatment (zero costs),

- 48 subjects participated in the low cost treatment,

- 48 played the high-cost treatment.

A summary of these treatments is shown in Table 2 . 
Table 2: Summary of treatments \& observations (replications)

\begin{tabular}{|c|c|c|c|c|c|}
\hline & sessions & rounds & repetitions & societies & observations \\
\hline$c=0$ & 5 & 5 & 0 & 25 & 200 \\
$c=5$ & 6 & 5 & 1 & 60 & 480 \\
$c=20$ & 6 & 5 & 1 & 60 & 480 \\
\hline
\end{tabular}

\subsection{Myopic and strategic behaviour}

Before starting with the analysis of the data we obtained from the experiments, we precisely explain under which circumstances "to stay" or "to move" has a clear meaning. The above Theorem holds that there are four types of behaviour in equilibrium. We describe these types in what follows.

The first one, Type I, is the behaviour followed by players 5 and 6 . Given the history $(\underline{1}, \underline{2}, \underline{3}, \overline{4})$, players 5 and 6 are already happy as a result of player 4's action. We can say that their best response, "to stay", is equivalent to what they would have done in the Schelling framework. In other words, both players 5 and 6 would play the same action, regardless of whether they were playing a myopic response or the SPE response. In both cases, they stay in their initial position. Therefore, in cases where players do not need to move it is not possible to distinguish whether they are playing the linear Schelling model or the sequential Schelling dynamics model.

The second type of behaviour is performed by players 1, 2, 3, and 7 . When these agents are called upon to play, no one is happy, but their best response is "to stay". Such behaviour implies that they have computed the best response to their associated subgame. ${ }^{20}$ Notice that in all of these nodes players play differently than when they act as myopic players. We call such behaviour Type II behaviour.

The third behaviour could appear in opposition to the above strategic behaviour. For instance, if player 7 played "to move" she would get a positive payoff $M$ because she ends up in a happy configuration. Nevertheless, such behaviour implies that player 7 did not compute her best response, taking into account the best response of player 8. We call such behaviour "myopic behaviour" or Type III behaviour.

Finally, we have Type IV individuals: players 4 and 8. Both players are unhappy and they decide "to move" in contrast with the above set of players $\{1,2,3,7\}$. This is so because their best action is "to move". In other words, should player 4

\footnotetext{
${ }^{20}$ The action "to stay" in such situations conveys a non-trivial computation.
} 
decide "to stay", the best response by the rest of the players would never generate a configuration with player 4 in a happy situation.

The four types described above are summarized in Table 3:

Table 3: Subject types

\begin{tabular}{|c|c|c|c|c|}
\hline Type & Initial & Best-Response & Myopic & Action \\
\hline I & Happy & Stay & Stay & Stay \\
II & Unhappy & Stay & Move & Stay \\
III & Unhappy & Stay & Move & Move \\
IV & Unhappy & Move & Move & Move \\
\hline
\end{tabular}

Although all players play their best response in equilibrium, we specially distinguish the set of Type II players $(\{1,2,3,7\})$, that we call strategic players. We emphasize that the action "to stay" played by unhappy players demonstrates ${ }^{21}$ the ability to compute the best response in the current subgame.

Formally,

Definition 1 (Type II, unhappy-strategic): Given history $h$, we define as strategic behaviour those unhappy $i$ who play their best-response "to stay" in $\Gamma_{h}$.

Definition 2 (Type III, unhappy-myopic): Given history $h$, we define as $m y$ opic behaviour those unhappy $i$ who play "to move" instead of their bestresponse "to stay" in $\Gamma_{h}$.

We should emphasize that there may exist another type of behaviour. Irrational subjects may choose to move in a situation in which they are already happy (notType I) or subjects who being not happy choose "to stay" when they do not have any chance of being happy unless they move ${ }^{22}$ (not-Type IV). Formally,

Definition 3 (Type $\neg \mathbf{I}$ and $\neg \mathbf{I V}$ ): Given history $h$, we define as irrational behaviour those happy (unhappy) $i$ who play "to move" ("to stay") when both their myopic and best response is "to stay" ("to move") in $\Gamma_{h}$.

Let us now focus on how the emergence of unhappy-strategic (Type II) versus unhappy-myopic (Type III) behaviour may affect the final outcome configuration.

\footnotetext{
${ }^{21}$ Theoretically, the rule of thumb "never move" could also explain the behaviour of Type II individuals in our study. However, we exclude this explanation as in our experiment we do not find any individual following that rule.

${ }^{22} \mathrm{An}$ individual follows the rule of thumb "never move" if she is categorized as Type II and notType IV at the same time. We do not find subjects fulfilling both conditions in our experiment.
} 
We have characterized all possible histories of length $i-1$ such that player $i$ faces the above dilemma: strategic versus myopic response. At each possible decision node of the above family of histories, agent $i$ should play "to stay" as her best response and therefore player $i$ declares herself to be Type II, unhappy-strategic. However, we also consider the other action, "to move", where player $i$ reveals herself to be Type III, unhappy-myopic. We can distinguish between both types since there is a one-to-one correspondence between actions and types, Types II and III, for the above family of histories. We assume that regardless of the action taken by player $i$, the remaining players $(i+1, \ldots, 8)$ play their subgame best response. A complete characterization is provided in Table $\mathrm{C}$ of Appendix $\mathrm{C}$.

The two following conclusions emerge from the above characterization. The first one is that in the absence of Type II players (row $4^{*}$ in Table $\mathrm{C}$, Appendix $\mathrm{C}$ ) the Schelling full segregation outcome will always emerge.

The second conclusion makes our results substantially different from the Schelling outcome. In the presence of one Type II player the Schelling full segregation outcome would emerge if and only if player 1 is strategic and players 2, 5 and 8 are myopic. See row $5^{*}$ in Table C, Appendix C.

Hence, the existence of strategic players may play a crucial role in the prevalence of Schelling predictions. Moreover, the emergence of irrational players Type $\neg$ I and $\neg$ IV may alter the previous idea. Actually, both types of behaviour enhance less segregated societies. However, this is not conclusive regarding the final configuration as the irrational action of player $i$ at node $k$ could be balanced out by the actions of subsequent players.

\subsection{Results}

This section is divided in three parts: first, we show the level of segregation achieved in each society within the different treatments; second, we present the fraction of individuals playing strategically across experiments; finally, we combine strategic behaviour and society segregation. We will show experimental evidence for both face-to-face and lab sessions.

\subsubsection{Segregation}

To explore this inquiry, we analyze the type of outcome achieved in each clock. As noted in the previous sections our experiment provides us with 138 societies ( 25 for $c=0$ treatment; 60 for $c=5$; and, 53 for $c=20$ ). In Figure 4 below, we show 
the fraction of these societies that achieve a fully segregated outcome, as defined by Schelling, that is, BBBBWWWW, as compared to the fraction where this outcome is not achieved.

While the BBBBWWWW outcome appears in $60 \%$ of the $c=0$ societies, this fraction notably decreases to $44.07 \%$ in societies with small moving costs $(c=5)$, although the difference between these two proportions is not statistically significant $(t=1.33, p=0.18)^{23}$. However, the fraction of fully segregated outcomes is significantly smaller $(t=1.74, p=0.08)$ for societies with large moving costs $(c=20)$, $38.8 \%$, as compared with societies without moving costs $(c=0)$. Further, this result is reinforced when we make an overall comparison of $c=0$ vs. $c>0$. We also obtain a significant reduction $(t=1.69, p=0.09)$ of fully segregated outcomes in the treatment with moving costs as compared to the costless treatment.

\section{Cost $=0$}

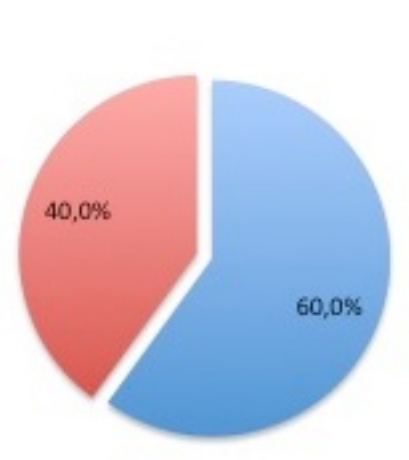

Cost $=5$

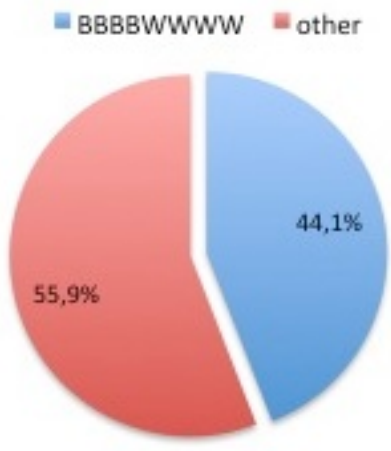

\section{Cost $=\mathbf{2 0}$}

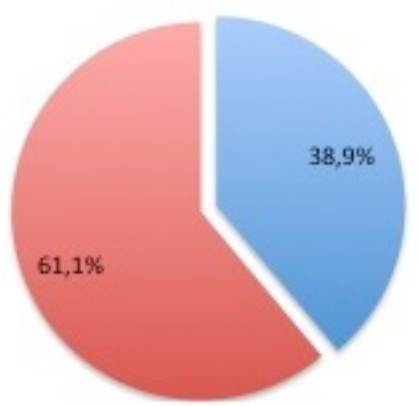

Figure 4: Fraction (\%) of BBBBWWWW outcomes across treatments.

Now we compare the results shown in Figure 4 with those we found in the lab replications explained in Table 2 :

- $c=0: 76.0 \%$ full segregation (19 out of 25 societies)

- $c=5: 53.3 \%$ full segregation (32 out of 60 societies)

- $c=20: 35 \%$ full segregation (21 out of 30 societies)

Lab results go in parallel to the face-to-face game: without costs the emergence of Scheling output is huge ${ }^{24}$ while under positive costs the BBBBWWWW outcome

\footnotetext{
${ }^{23}$ Non parametric tests report identical results.

${ }^{24}$ The $76 \%$ full segregation for zero costs is statistically different to $53.3 \%(p-$ value $=0.052)$,
} 
becomes less likely to emerge. ${ }^{25}$ We find a negative relationship between the size of moving costs and the share of segregated outcomes, although it is not always statistically significant.

All in all, we summarize the findings shown along this subsection as follows:

Result 1: Under positive moving costs, the BBBBWWWW outcome is (significantly) less likely to emerge.

What we learn from this result is that Schelling prediction is very powerful for costless environments but it is less precise for situations where moving costs are relevant. In fact, what Result 1 says is that full segregation is less likely to emerge in societies where moving is costly. Today, in most of the cities around the word, moving is extremely costly and time consuming. Although our experiment is just a "small and artificial" replication of the real world it is also true that our results show, in a certain way, what we see all around the world: segregated and not segregated cities.

Result 1 talks about segregation, that is, about aggregated behaviour. The next subsection is devoted to study how subjects behave individually.

\subsubsection{The emergence of strategic players}

Once we have checked that Schelling's outcome is less likely to emerge in costly moving societies (Result 1), we turn to analyze how moving costs affect individual behaviour. In fact, only under costly environments can we assess the appearance of strategic behaviour.

Using the information provided by each clock, we compute, for all empirical histories, ${ }^{26}$ those cases where unhappy subjects face the dilemma either "to stay" or "to move", when the situation is not trivial (Type II, unhappy-strategic versus Type III, unhappy-myopic, see definitions in page 20). More specifically, after the actions played by the previous individuals, each unhappy agent calculates her best response before taking any action. There are two possibilities, either the best response is "to move" or "to stay". If the best response is "to move", this implies that the unhappy

and to $35 \%(p-$ value $=0.001)$. Further, the $53.3 \%$ of fully segregated outcomes is statistically different to $35 \%(p-$ value $=0.044)$.

${ }^{25}$ As the face-to-face experiment, when we make an overall comparison of $c=0$ vs. $c>0$ we get a significant reduction $(t=1.64, p=0.10)$

${ }^{26}$ All the empirical histories we obtain in the experiment are reported in Appendix B. In each history we underline who behaves strategically and who deviates from such behaviour. 
agent anticipates the best actions of subsequent players, and only if she moves will she finish in a happy configuration. Nevertheless, if she computes the best response of the remaining players and she finishes in a happy configuration even if she does not move, then, "to stay" becomes her best response since she does not need to give up her initial endowment ( 5 or 20 Euros). However, we do not find this feature in the 0 cost setting since subjects are indifferent between both actions.

We should point out that although we have 904 observations (see last column of Table 1) only 320 of them are informative about the type of behaviour we are interested in (either myopic or strategic). ${ }^{27}$

Along the $c>0$ treatments we are able to find 128 strategic movements (Type II). Figure 5 shows the presence of strategic players across the 113 societies we obtained $(60+53)$. The $x$-axis represents the proportion of Type II players $(0,1,2$ or $3+)$ among the 8 participants of a clock. ${ }^{28}$

Figure 5: Distribution of strategic players across societies

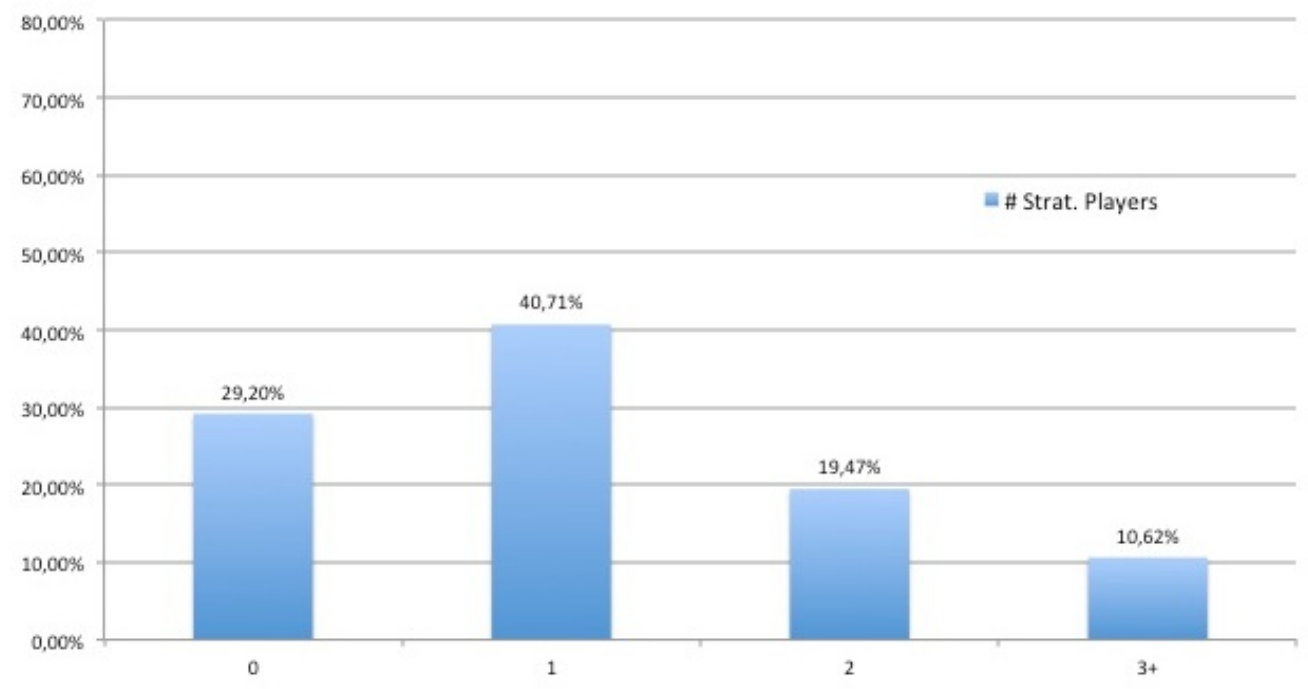

The results in Figure 5 show the striking result that only $29.20 \%$ of the societies are completely free of strategic players. In other words, in most of the outcomes $(70.80 \%)$ we observe at least one player behaving strategically. In fact, in one third

\footnotetext{
${ }^{27}$ As we have already mentioned, we are unable to confirm strategic behaviour for Type I and Type IV, see Table 3 .

${ }^{28}$ Recall that it is impossible to have more than 4 Type II players.
} 
of the societies there are 2 or more individuals who do not behave according to Schelling myopic rule.

It is also necessary to analyse what occurs in the lab, where anonymity is preserved. We do find quite similar results in the lab than in the face-to-face. Specifically:

- $30.83 \%$ of the societies have 0 strategic players vs. $29.20 \%$ in Figure 5,

- $35.0 \%$ of the societies have 1 strategic player vs. $40.71 \%$ in Figure 5,

- $21.67 \%$ of the societies have 2 strategic players vs. $19.47 \%$ in Figure 5 ,

- $12.5 \%$ of the societies have $3+$ strategic players vs. $10.62 \%$ in Figure 5.

Therefore it is reasonable to say that the face-to-face experiment gives the same results as the computerized one. Therefore we may conclude that,

Result 2: Under positive moving costs, the majority of the societies has at least one strategic player.

Result 2 indicates that the existence of positive costs make subjects more aware of playing strategically. We get this result both in the face-to-face $(70.80 \%)$ and lab experiments $(69.17 \%)$.

As a final remark we want to emphasize what we can learn from our experimental setting. We can check if a experimental subject fails at least once playing myopically. It is also true that subjects who play myopically in our experiment (given the position, history, etc.) might play strategically in other environments. In sum, we can say that the number of strategic players we are able to uncover is a lower bound.

\subsubsection{Strategic behaviour and segregation}

Our theoretical model indicates that just one strategic player might be enough to reverse the Schelling output. Our experimental data show that: i) Schelling configuration is not always achieved (see Result 1); and, ii) Strategic behaviour is not rare but, on the contrary, quite abundant in the treatments with moving costs (see Result 2).

Now, we check if Schelling's prediction is related to the number of strategic players within a particular society. We now combine societal segregation across treatments and individual behaviour, that is, we explore whether the final level of segregation achieved in each clock depends on the number of strategic players within that clock. 
Our results indicate that the emergence of strategic players substantially changes the final segregation outcome achieved. Figure 6 shows the proportion of $0,1,2$ and $3+$ strategic players among those societies achieving full segregation, i.e., the BBBBWWWW outcome. ${ }^{29}$

Figure 6: Proportion of societies with strategic players and BBBBWWWW configuration

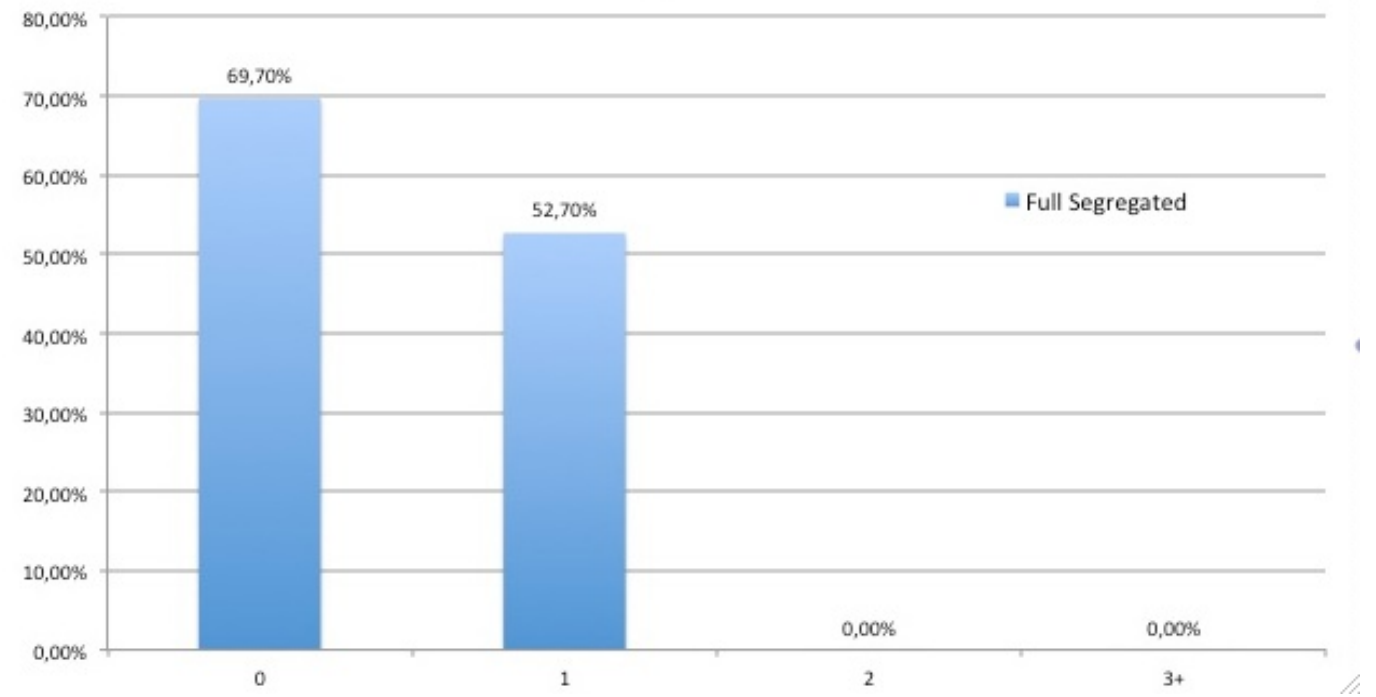

Figure 6 indicates that in the absence of strategic players the complete segregation outcome is reached in almost $69.70 \%$ of the cases. One single strategic player decreases this fraction to $52.70 \%$. Finally, having 2 or more strategic players eliminates the possibility of a fully segregated society. Hence, the Schelling fully segregated outcome seems to be significant and negatively related with the existence of Type II (unhappy-strategic) players within a clock. The proportion of full segregated configurations with 1, 2 or $3+$ strategic players is significantly smaller than that without strategic players $(t=3.89$ and a $p$-value of 0.0001$) .{ }^{30}$

The lab replication shows similar results but not identical. In fact, the proportion of societies with the fully segregated outcome when the number of strategic player is $0,1,2$ and $3+$ is $58.18 \%, 46.52 \%, 27.77 \%$ and $17.85 \%$, respectively.

Hence, like in Figure 6, there is a negative relationship between the number of strate-

\footnotetext{
${ }^{29}$ Recall that the analysis is restricted to treatments with moving costs.

${ }^{30}$ The difference between $69.70 \%$ and $52.70 \%$ is only significant at a $11.6 \%$. The difference between $69.70 \%$ and $0 \%$ (when we have 2 and $3+$ strategic players) is significant at any level.
} 
gic players and the likehood of full-segregated societies. However, we do not find support for the idea that a certain number of strategic players denies the existence of BBBBWWWW configurations.

These results can be summarized as:

Result 3: The full segregated outcome vanishes as the number of strategic players increases.

As shown in figure 5, we only had $30 \%$ of societies with none strategic player, therefore we can conclude that the Schelling prediction of full segregation significantly gets reduced in environments with moving costs.

\subsection{Discussion of the experimental results}

Using an experiment in a simplified environment, this paper shows that not all subjects play myopically when there are moving costs associated to their decisions of moving but, in contrast, a relevant fraction of them anticipate what the other subjects are going to do. This type of strategic behaviour was not considered in the most general model of spatial segregation, the model proposed by Thomas $\mathrm{C}$. Schelling (1971). Recall that it is the cost of traveling what makes the critical distinction between myopic individuals and those who are not.

But the crucial result is not the emergence of this type of behaviour per se but how the presence of this type of players alters the final outcome achieved in this artificial society: the higher the presence of them the smaller the probability of a fully segregated society. However, it is important to mention that the Schelling outcome appears in a significant number of cases.

In other words, our experiment shows that subjects respond to incentives but not all of them respond in an analogous way. In parallel, the emergence of strategic players has consequences on the final outcome, but once again, the resulting societies are not always equally distributed.

In summary, our results indicate that individuals react in different ways and that heterogeneous individuals can reach both segregated environments and not segregated ones. 


\section{CONCLUDING REMARKS}

The relevance of this paper is twofold. First, we provide an extension of the Schelling linear model where subjects face costly decisions and, as a consequence, strategic playing emerges. We show that this variation affects the basic result of the model by moving from full segregation to clustering. Second, we experimentally test the prediction of the original Schelling model and the extended model we propose. That is, we develop a geographical experimental setup where we check whether experimental subjects play according to the predicted Schelling myopic behaviour or, in sharp contrast, they decide as strategic players.

This paper contains certain features that support the development of an interesting research agenda. The most obvious example is that we provide a new experimental setup to explore the role of locational issues on strategic behaviour. Given that subjects learn easily how to play in this environment, the emergence of strategic behaviour is not rare. Moreover, we find that visual experimental setups make subjects more aware of their rivals' strategic playing.

We must recall that considering different types of behaviour was one of the main reasons why we undertook this study. Specifically, we were interested in exploring how differences among players may have an effect on economic outcomes. Our simplified representation of the society is useful in analyzing how subjects play these "sorted games". ${ }^{31}$ We plan to extend our research agenda to improve the applicability of our work, including ethnic segregation with income differentiation. In the same vein, and in line with some works in public policy, we are working on Schelling dynamic models with taxation concerning situations where the state designs policies to achieve more desirable configurations.

\section{REFERENCES}

[1] Benito, Juan M., Pablo Brañas-Garza, Penélope Hernández, and Juan A. Sanchis. 2011. "Sequential versus Simultaneous Schelling Model: Experimental Evidence". Journal of Conflict Resolution, 55 (1), 33-59.

\footnotetext{
${ }^{31}$ Julie Berry Cullen, Brian A. Jacob and Steven Levit (2006) is an excellent example of how random (public lottery) versus endogenous (parental choice) sorting may have an effect on child academic performance.
} 
[2] Card, David, Alexandre Mas, and Jesse Rothstein. 2008. "Tipping and the Dynamics of Segregation." Quarterly Journal of Economics. 126(1): 177-218.

[3] Clark, William A. V., and Mark Fossett. 2008. "Understanding the social context of the Schelling segregation model." Proceedings of the National Academy of Science of the United States of America, 105(11): 14109-4114.

[4] Cullen, Julie Berry, Brian A. Jacob, and Steven Levitt. 2006. "The Effect of School Choice on Participants: Evidence from Randomized Lotteries". Econometrica, 74(5): 1191-1230.

[5] Cutler, David M., Edward L. Glaeser, and Jacob L. Vigdor. 1999. "The Rise and Decline of the American Ghetto." Journal of Political Economy, 107(3): 455-506.

[6] Follain, James R., and Stephen Malpezzi. 1981. "Another look at racial differences in housing prices." Urban Studies, 18(2): 195-203.

[7] Foster, Dean P., and H. Peyton Young. 1990. "Stochastic Evolutionary Game Dynamics." Theoretical Population Biology, 38(2): 219-232.

[8] Karni, E., and D. Schmeidler. 1990. "Fixed preferences and changing tastes", American Economic Review 80(2), 262-267.

[9] King, A. Thomas, and Peter Mieszkowski. 1973. "Racial discrimination, segregation, and the price of housing." Journal of Political Economy, 81(3): 590-606.

[10] Kling, Jeffrey R., Jeffrey B. Liebman, and Lawrence F. Katz (2007), "Experimental Analysis of Neighbourhood Effects", Econometrica 75, 83-119.

[11] Miltaich, I., and E. Winter. 2002. "Stability and Segregation in Group Formation", Games and Economic behaviour 38, 318-346.

[12] Osborne, Martin J., and Ariel Rubinstein. 1996. A Course in Game Theory. Cambridge, Massachusetts: The MIT Press.

[13] Pancs, Romans, and Vriend Nicolaas J. 2007. "Schelling's spatial proximity model of segregation revisited." Journal of Public Economics, 91(1): 1-24.

[14] Rubinstein, Ariel. (2007). Instinctive and Cognitive Reasoning: A Study of Response Times, Economic Journal, 117 (2007), 1243-1259.

[15] Schelling, Thomas C. 1969. "Models of Segregation." American Economic Review, Papers and Proceedings, 59(2): 488-493. 
[16] Schelling, Thomas C. 1971. "Dynamic Models of Segregation." Journal of Mathematical Sociology, 1(2): 143-186.

[17] Young, H. Peyton. 1998. Individual Strategy and Social Structure: An Evolutionary Theory of Institutions. Princeton, NJ: Princeton University Press.

[18] Young, H .Peyton. 2001. The Dynamics of Conformity. In S.N. Durlauf \& H.P. Young (Eds.), Social Dynamics, 132-153. Cambridge, Massachusetts: The MIT Press.

[19] Young, H. Peyton. 2009. "Innovation Diffusion in Heterogeneous Populations: Contagion, Social Influence, and Social Learning." American Economic Review, 99(5): 1899-1924.

[20] Zhang, Junfu. 2004. "Residential Segregation in an All-integrationist World." Journal of Economic behaviour \& Organization, 54(4): 533-550.

\section{Appendix A}

First, let's formally describe the sequential game denoted by $\Gamma^{n}=\left(\mathbf{N}=\{1,2, \ldots, N\}, A_{i}=\right.$ $\left.\{\underline{i}, \bar{i}\}_{i \in \mathbf{N}},\{K\}_{1, \ldots, \sum_{i=0}^{N} 2^{i}},\{Z\}_{2^{N-1}, \ldots, 2^{N}-1}, u_{i}: \prod_{i=1}^{N} A_{i} \rightarrow \mathbb{R}\right)$ where

- $\mathbf{N}=\{1,2, \ldots, N\}$ is the set of players.

- $A_{i}=\{\underline{i}, \bar{i}\}$ is the set of actions for each player such that $\underline{i}$ means that player $i$ stays at her initial location and $\bar{i}$ that player $i$ moves to the nearest space with someone of her same type.

- $K$ is the set of nodes and $Z$ is the set of terminal nodes. Notice that all nodes in $K-Z$ are information sets for only one player.

- The map $i: \mathbb{H}=K-Z \rightarrow\{1,2, \ldots, N\}$ where $I(k)=i$ such that $\sum_{j=0}^{i-1} 2^{j} \leq$ $k \leq \sum_{j=0}^{i} 2^{j}-1$ determines the agent playing at this node.

- The set of terminal nodes $Z=\left\{\sum_{j=0}^{N-1} 2^{j}+1, \ldots, \sum_{j=0}^{N} 2^{j}\right\}$ where payoffs $\left\{u_{i}\right\}_{i \in \mathbf{N}}$ are realized.

Then, for each $s$ in $K-Z$ an equilibrium strategy should specify the best action for player $i(s)$ who plays at $s$. Let us fix $h_{s} \in A_{1} \times \ldots \times A_{i(s)-1}$ as the unique history from the root node reaching $s$ and denote by $\Gamma_{h_{s}}^{n}$ the subgame starting at $s$.

The payoff for player $i$ depends on her action and the final configuration reached after all players have played. If her action was to stay and at the end of game the 
number of her neighbours like her is at least $m$ then she gets $M+C$. Otherwise she only gets $C$. If her action was to move and she reached a position with at least $m$ neighbours like her then her payoff is $M$. Otherwise, she will obtain 0 . Notice that she will never be indifferent between both actions, to move and to stay. Therefore the property of no indifference holds. In other words, $\Gamma^{n}$ is a finite generic extensive game. Consider any possible initial configuration of $n$ individuals of type 0 or 1 . This configuration can be viewed as a sequence in $\{0,1\}^{n}$. Next theorem asserts the existence and unicity of a SPE for $\Gamma^{n}$ independent of the initial condition. Moreover the explicit equilibrium strategy for $n=8, d=m=1$ and the initial configuration $\{B, W, B, W, B, W, B, W\}$ is provided.

Theorem 1. - There exists a unique subgame perfect equilibrium in the extensive game $\Gamma^{n}$.

- For $d=m=1$ and the initial configuration $\{B, W, B, W, B, W, B, W\}$, the final configuration $\{B, B, W, W, B, B, W, W\}$ is the consequence of the unique equilibrium path $(\underline{1}, \underline{2}, \underline{3}, \overline{4}, \underline{5}, \underline{6}, \underline{7}, \overline{8})$.

Proof. $i$ ) The existence of a pure Nash equilibrium of the extensive game $\Gamma^{n}$ is a consequence of Zermelo's Theorem since $\Gamma^{n}$ is a finite game. Moreover by Osborne and Rubinstein (1996), page 100, this game verifies the non indifference property for any two terminal nodes. Therefore, all the SPE are equivalent in payoffs. As the game is generic then the result holds.

Recall that $d \geq m$. If the number of players of each type is at least $m+1$ then the final society is always happy for any initial configuration. This is so since there exist at least a path over the $2^{n}$ possible paths of actions of the extensive game with a cluster of these $m+1$ players one after the other. Each player has $m$ neighbours like her in a neighbourhood of ratio $d$.

The existence of a pure Nash equilibrium of the extensive game associated to the initial configuration $\{B, W, B, W, B, W, B, W\}$ holds since it is a particular case of the above case. At node $s$ of the extensive game, a player, say $i_{s}$, will play her best response given history $h_{s}$ and considering that the rest of the players will play their best response. Therefore, in order to describe the best strategy for each player, we distinguish two sets of histories: those in which player $i_{s}$ is already happy and those that do not verify the happiness condition. Notice that for the first set of histories, the best response for player $i_{s}$ is always "to stay" since her payoff is $M+C$ greater than $M$.

1. The analysis starts with the last player; player 8 .

(a) The histories of length 7 where player 8 is happy are: 
- $h(s)=(*, *, *, *, *, \overline{6}, *)$

- $h(s)=(*, *, *, *, \underline{5}, \underline{6}, \overline{7})$

- $h(s)=(\overline{1}, \underline{2}, *, *, *, *, *)$

where the symbol $*$ denotes any possible strategy for the player who has to play at this position. As we already noted, her best-response is "to stay" $\underline{8}$

(b) Consider now the histories where player 8 is unhappy:

- $h(s)=(\overline{1}, \overline{2}, *, *, *, \underline{6}, \underline{7})$

- $h(s)=(\underline{1}, *, *, *, *, \underline{6}, \underline{7})$.

- $h(s)=(\overline{1}, \overline{2}, *, *, \overline{5}, \underline{6}, \overline{7})$

In all these cases, as player 8 is the last player, her best response is "to move" $\overline{8}$.

2. To compute the strategy of player 7 , we take into account both the history and the best reply of player 8 given the two possible actions of player 7 .

(a) Player 7 is happy in the following cases:

- $h(s)=(*, *, *, *, \overline{5}, *)$

- $h(s)=(*, *, *, \underline{4}, \underline{5}, \overline{6})$

Her best response is "to stay" $\underline{7}$.

(b) Player 7 is unhappy if her history is $(*, *, *, *, \underline{5}, \underline{6})$. As the action "to stay" is dominant over the action "to move", we check if "to stay" is her best response given the corresponding best response of player 8 :

- The two following histories $h(s)=(\overline{1}, \overline{2}, *, *, \underline{5}, \underline{6})$ and $h(s)=(\underline{1}, *, *, *, \underline{5}, \underline{6})$ have in common that for action $\underline{7}$, the best response for player 8 is "to move". Consequently, at the final configuration, player 7 becomes happy given the best response of player 8 . Therefore, in those cases, the best response for player 7 is "to stay", $\underline{7}$.

- Nevertheless, the history $h(s)=(\overline{1}, \underline{2}, *, *, *, \underline{6})$ with action $\underline{7}$ presents a path for player 8 within a best response of $\underline{8}$. Therefore player 7 has to move in order to end up with at least one neighbour like her. We conclude that her best response is $\overline{7}$.

To summarize, depending on the action of player 1 and 2 , the path generated by the best responses of players 7 and 8 is either $(\underline{7}, \overline{8})$ or $(\overline{7}, \underline{8})$.

3. Following the same argument as before, the best response for player 6 depends on her history of length 5 and the actions that player 7 and 8 will play afterwards.

(a) Player 6 is happy if:

- $h(s)=(*, *, *, \overline{4}, *)$

- $h(s)=(*, *, \underline{3}, \underline{4}, \overline{5})$

thus her best response is "to stay", $\underline{6}$. 
(b) Player 6 is unhappy in the following histories of length 5 :

- $h(s)=(\overline{1}, \underline{2}, *, \underline{4}, \underline{5})$. In this situation, player 8 is happy since player 1 moved. Using the conclusion obtained above for players 8 and 7 , player 6 anticipates their best response $(\overline{7}, \underline{8})$. Therefore, giving the movement of player 7 , the best response for player 6 will be "to stay", $\underline{6}$.

- $\bar{h}(s)=(\overline{1}, \underline{2}, \overline{3}, \underline{4}, \overline{5})$. As in the above case, we can conclude that she will decide "to stay", $\underline{6}$.

- $h(s)=(\underline{1}, *, *, \underline{4}, \underline{5}),(\overline{1}, \overline{2}, *, \underline{4}, \underline{5}), h(s)=(\underline{1}, *, \overline{3}, \underline{4}, \overline{5})$ and $h(s)=$ $(\overline{1}, \overline{2}, \overline{3}, \underline{4}, \overline{5})$. In all these cases, player 8 is unhappy. Now player 6 anticipates that player 7 and 8 will play $(\underline{7}, \overline{8})$. Consequently, player 6 has "to move" to end up with at least one neighbour like her.

As a consequence, depending on the action of player 1, "to stay" or "to move", the path generated by the best response of players 6,7 and 8 are $(\overline{6}, \underline{7}, \underline{8})$ and $(\underline{6}, \overline{7}, \underline{8})$, respectively.

4. The history of length 4 and the best strategies for players 6,7 and 8 dictate the best answer for player 5 .

(a) If 5 is happy, the histories of length 4 are:

- $h(s)=(*, *, \overline{3}, *)$

- $h(s)=(*, \underline{2}, \underline{3}, \overline{4})$

with her best response being "to stay", $\underline{5}$.

(b) If 5 is unhappy, we consider:

- $h(s)=(\overline{1}, \underline{2}, \underline{3}, \underline{4})$ where player 5 will anticipate the action profile $(\underline{6}, \overline{7}, \underline{8})$. Hence her best response is "to move", $\overline{5}$.

- $h(s)=(\overline{1}, \overline{2}, \underline{3}, \overline{4}), h(s)=(\underline{1}, \overline{2}, \underline{3}, \overline{4})$ have in common that player 8 is not happy. Therefore player 5 anticipates $(\overline{6}, \underline{7}, \underline{8})$ and plays "to stay", $\underline{5}$, as her best response.

Hence the actions of players 1 and 2 condition the action of player 8 (either "to stay" or "to move") and the paths generated by the best response of players $5,6,7$ and 8 are $(\underline{5}, \overline{6}, \underline{7}, \underline{8})$ or $(\overline{5}, \underline{6}, \overline{7}, \underline{8})$, respectively.

5. Following the same process, player 4 considers:

(a) If 4 is happy, the histories of length 3 are:

- $h(s)=(*, \overline{2}, *)$

- $h(s)=(\underline{1}, \underline{2}, \overline{3})$

then her best response is "to stay", $\underline{4}$

(b) If 4 is unhappy, then the histories corresponding to such a situation are:

- $h(s)=(\overline{1}, \underline{2}, \underline{3})$ where player 4 anticipates $(\overline{5}, \underline{6}, \underline{7}, \underline{8})$ and her best response is "to stay", $\underline{4}$. 
- $h(s)=(\underline{1}, \underline{2}, \underline{3})$ where player 8 is not happy and therefore player 4 anticipates $(\underline{5}, \overline{6}, \underline{7}, \underline{8})$, being her best response "to move", $\overline{4}$.

- $h(s)=(\overline{1}, 2, \overline{3})$ where player 8 and player 5 are both happy. Following the subgame perfection equilibrium path of the corresponding subtree, player 4 anticipates $(\underline{5}, \underline{6}, \overline{7}, \underline{8})$. Her best response is then "to move", $\overline{4}$.

In any situation where player 4 is not happy, she has to move generating the path $(\overline{4}, \underline{5}, \underline{6}, \underline{7}, \overline{8})$.

6 . The case for player 3 is the following:

(a) If 3 is happy, the histories of length 2 are:

- $h(s)=(\overline{1}, *)$

- $h(s)=(\underline{1}, \overline{2})$

then her best response is "to stay", $\underline{3}$.

(b) If 3 is unhappy, then the unique history to be considered is:

- $h(s)=(\underline{1}, \underline{2})$ where player 3 will anticipate $(\overline{4}, \underline{5}, \underline{6}, \underline{7}, \overline{8})$. Thus her best response is "to stay", $\underline{3}$.

Therefore, the best response for player 3 in any situation is "to stay", $\underline{3}$.

7. For the case of player 2, we have only two histories:

(a) If 2 is happy:

- $h(s)=(\overline{1})$

then her best response is "to stay", 2 .

(b) If 2 is unhappy, then the unique history to consider is:

- $h(s)=(\underline{1})$ where player 2 anticipates $(\underline{3}, \overline{4}, \underline{5}, \underline{6}, \underline{7}, \overline{8})$, thus her best response is "to stay", $\underline{2}$, because player 8 will move to the space close to her.

The above situation therefore means that the best reply for player 2 is "to stay", $\underline{2}$.

8. The last case corresponds to player 1 with only one case:

(a) 1 is unhappy at the initial state so she will anticipate $(\underline{2}, \underline{3}, \overline{4}, \underline{5}, \underline{6}, \underline{7}, \overline{8})$.

Her best response is "to stay", $\underline{1}$, because player 8 will move to the space.

Given the above situation, the best reply for player 1 is "to stay", $\underline{1}$.

The unique equilibrium path of $\Gamma$ is $(\underline{1}, \underline{2}, \underline{3}, \overline{4}, \underline{5}, \underline{6}, \underline{7}, \overline{8})$ with a final circle configuration of players 18235467 generating a society of $B W W B B W W B$. Hence the result holds.

\section{Appendix B}

This appendix is devoted to studying some particular paths generated by the subgame best response after a specific history. Specifically, the extensive game has many 
paths that do not verify the subgame perfect criterion. Any of them establishes at least one player who plays off the equilibrium path, that is, players who are not Type II. Nevertheless, these choices may reach either a happy society where all players end up with at least a neighbour like them or not. The goal of the following discussion is to study those cases that arise in our experimental data by characterizing player types.

Let us start with the second row of Table B in this Appendix, $(\overline{1}, \underline{2}, \underline{3}, \underline{4}, \overline{5}, \underline{6}, \underline{7}, \underline{8})$. This configuration appears in our empirical data with a frequency of $3.33 \%$. The lemma below states the SPE of the subgame after the action of player 1 . In the particular case where player 1 is Type III, the generated tree denoted by $\Gamma_{\overline{1}}$ can be solved for the remaining players using the subgame perfect criterion. This allows us to determine each player's type in the path obtained.

Lemma 2. If player 1 plays $\overline{1}$, then the $S P E$ for $\Gamma_{\overline{1}}$ is $(\underline{2}, \underline{3}, \underline{4}, \overline{5}, \underline{6}, \underline{7}, \underline{8})$. The corresponding output society is $B B W W B B W W$.

Proof. As player 1 moves, player 8, 2 and 3 become happy. We can conclude that the best response for all of them is "to stay". Let us check what is the best response for players 4, 5, 6 and 7 .

Player 4 will react against the history of length $3:(\overline{1}, \underline{2}, \underline{3})$. By the proof of the main theorem, we know that player 4 will anticipate the action profile for players 5,6 , 7 and 8: $(\overline{5}, \underline{6}, \underline{7}, \underline{8})$. Therefore, her best response is "to stay", $\underline{4}$. The final output configuration is obtained by the path $(\overline{1}, \underline{2}, \underline{3}, \underline{4}, \overline{5}, \underline{6}, \underline{7}, \underline{8})$, thus generating the society $B B W W B B W W$.

Given the above lemma, we can conclude that player 1 is Type III and player 4 is Type II. Nevertheless, given that the remaining players are Type I, we are not able to distinguish between strategic behaviour and myopic behaviour.

Consider now the following path: $(\underline{1}, \overline{2}, \underline{3}, \underline{4}, \underline{5}, \overline{6}, \underline{7}, \underline{8})$. This path corresponds to the fourth row in Table B. In this case, player 1 is of Type II, but player 2 is of Type III. The next lemma states the subgame path of the corresponding subtree after the history $(\underline{1}, \overline{2})$.

Lemma 3. If player 1 plays 1 and player 2 plays $\overline{2}$, then the $S P E$ for $\Gamma_{1, \overline{2}}$ is $(\underline{3}, \underline{4}, \underline{5}, \overline{6}, \underline{7}, \underline{8})$.

Proof. After the action of player 2, both player 3 and player 4 become happy. Following the proof of the main result, we can check that if player 5 decides "to stay" it is because she anticipates the best responses of players 6,7 , and $8:(\overline{6}, \underline{7}, \underline{8})$. With this path, player 5 becomes happy and thus her best response is "to stay". 
In this case, player 5 plays $\underline{5}$ even when she is not happy after her history $h_{5}=$ $(\underline{1}, \overline{2}, \underline{3}, \underline{4})$. This yields Type II behaviour for player 5 . The final configuration of the final society is $B B W W B B W W$.

The last lemma in this section presents a variation of types and the consequent best response for an information node of a Type III player. This case is shown in the third row of Table B in this Appendix.

Fix the action of player $1, \overline{1}$. By lemma 2 , the corresponding subgame perfect path of the subtree $\Gamma_{\overline{1}}$ is $(\underline{2}, \underline{3}, \underline{4}, \overline{5}, \underline{6}, \underline{7}, \underline{8})$. In this case, player 4 is of Type II. What is the best response if player 4 were actually of Type III?

Lemma 4. If players 1, 2, 3 and 4 play $(\overline{1}, \underline{2}, \underline{3}, \overline{4})$, the $\operatorname{SPE}$ for $\Gamma_{(\overline{1}, \underline{2}, \underline{3}, \overline{4})}$ is $(\underline{5}, \underline{6}, \overline{7}, \underline{8})$.

Proof. Notice that player 8 is happy given the action of player 1 . Moreover, as player 4 is of Type III, she makes players 5 and 6 happy. Player 7 is therefore the last unhappy player. Following the proof of the main result, we can conclude that her best response is "to move".

In the above case, there are no Type II individuals. Moreover, a Type IV individual appears: player 7 . The final configuration is not a happy configuration: $W B B W W W B B$.

The following table shows the rest of the empirical cases ${ }^{32}$ in our experiment. The first column shows the path. The second one gives the players who deviate from the equilibrium path, i.e., Types III, $\neg$ I and $\neg$ IV. The third column enumerates the players engaging in strategic behaviour (Type II), while the last column presents the final configuration of the society.

\footnotetext{
${ }^{32} \mathrm{~A}$ non-rational deviation could be any movement after an immediate movement of your neighbour. Then, any path with two consecutive movements is not an equilibrium path and is not a best response for the last player. As we find some of these cases in our data, we study them one by one.
} 


\begin{tabular}{|c|c|c|c|}
\hline Play & TypesIII, $\neg I, \neg I V$ & TypeII & Outcome \\
\hline$(\underline{1}, \underline{2}, \underline{3}, 4, \underline{5}, \underline{6}, \underline{7}, 8)$ & & $1,2,3,7$ & $B B W W B B W W$ \\
\hline$(\mathbf{1}, \underline{\mathbf{2}}, \underline{\mathbf{3}}, \underline{\mathbf{4}}, \underline{\mathbf{6}}, \underline{\mathbf{7}}, \underline{\mathbf{8}})$ & 1 & 4 & BBWWBBWW \\
\hline$(\mathbf{1}, \underline{\mathbf{2}}, \underline{\mathbf{3}}, \underline{\mathbf{4}}, \underline{\mathbf{6}}, \overline{\mathbf{7}}, \underline{\mathbf{8}})$ & 1,4 & & $B B B B W W W W$ \\
\hline$(\underline{1}, \overline{2}, \underline{\mathbf{3}}, \underline{\mathbf{5}}, \overline{\mathbf{6}}, \underline{\mathbf{7}}, \underline{\mathbf{8}})$ & 2 & 1,5 & BBWWBBWW \\
\hline$(\underline{1}, 2, \underline{3}, \underline{\overline{4}}, \underline{6}, \underline{7}, \overline{8})$ & 2,5 & 1 & $B B B B W W W W$ \\
\hline$(\underline{1}, \underline{2}, \overline{3}, \underline{4}, \underline{5}, \overline{6}, \overline{7}, \underline{8})$ & 3 & 1,2 & BWWBBBWW \\
\hline$(\underline{1}, \underline{2}, \underline{3}, \underline{4}, \underline{6}, \overline{7}, \underline{8})$ & 7 & $1,2,3$ & $B B W B B W W W$ \\
\hline$(1, \overline{2}, \underline{3}, \underline{4}, \underline{\overline{5}}, \underline{7}, \underline{8})$ & 1,2 & 5 & $B B W W B B W W$ \\
\hline$(1,2, \underline{3}, \underline{4}, \overline{5}, \underline{6}, \underline{7}, \overline{8})$ & $1,2,5$ & & $B B B B W W W W$ \\
\hline$(1, \overline{2}, \underline{3}, \underline{4}, 5, \overline{6}, \underline{7}, \underline{8})$ & $1,2,5,6$ & & $B B W W B B W W$ \\
\hline$(\underline{1}, \underline{2}, \overline{3}, \underline{4}, \underline{5}, 6, \overline{7}, \underline{8})$ & 3,7 & 1,2 & $B W W B B W W B$ \\
\hline$(\underline{1}, \underline{2}, 3,4, \underline{5}, \underline{6}, \underline{7}, \overline{8})$ & 3,4 & $1,2,7$ & $B B W W B B W W$ \\
\hline$(\underline{1}, \underline{2}, 3,4, \underline{5}, \underline{6}, \overline{7}, \underline{8})$ & $3,4,7$ & 1,2 & $W W B W W B B B$ \\
\hline$(\underline{1}, \underline{2}, 3,4, \underline{5}, \underline{6}, 7, \overline{8})$ & $3,4,7,8$ & 1,2 & $W W B B W W B B$ \\
\hline$(\overline{1}, \underline{2}, \underline{3}, 4, \overline{5}, \underline{6}, \overline{7}, \underline{8})$ & $1,4,5$ & & $B B W W B B W W$ \\
\hline$(\underline{1}, \overline{2}, \underline{3}, \underline{4}, \overline{6}, \underline{7}, \underline{8})$ & $2,5,6$ & 1 & $B B W W B B W W$ \\
\hline$(\underline{1}, 2,3, \underline{4}, \underline{5}, 6, \underline{7}, \underline{8})$ & 2,3 & 1 & $B B W W B B W W$ \\
\hline$(\overline{1}, \overline{2}, \underline{3}, \underline{4}, \overline{5}, \underline{6}, \overline{7}, \underline{8})$ & $2,3,6,7$ & 1 & $B B W W B B W W$ \\
\hline$(1, \underline{2}, \underline{3}, \underline{4}, \underline{5}, \underline{6}, 7, \underline{8})$ & 1,5 & 4,6 & $B B W B W W W B$ \\
\hline$(1, \underline{2}, \underline{3}, \underline{\overline{5}}, \overline{\overline{6}}, \underline{7}, \underline{8})$ & $1,4,6$ & & $B B B W B W W W$ \\
\hline$(1, \underline{2}, \underline{3}, 4, \underline{5}, \underline{6}, \underline{7}, \underline{8})$ & $1,4,7$ & & $B B B W W B W W$ \\
\hline$(1, \underline{2}, \underline{3}, \underline{4}, \overline{5}, \underline{6}, \overline{7}, \underline{8})$ & 1,7 & 4 & $B B W W B W W B$ \\
\hline$(1, \underline{2}, \underline{3}, \underline{4}, \underline{5}, \overline{6}, \underline{7}, \underline{8})$ & $1,5,6$ & 4 & $B B W B B W W W$ \\
\hline$(1, \underline{2}, \underline{3}, \overline{4}, \overline{5}, \underline{6}, \underline{7}, \underline{8})$ & $1,4,5$ & & $B B W W B B W W$ \\
\hline$(1, \underline{2}, \overline{3}, 4, \underline{5}, \underline{6}, \overline{7}, \underline{8})$ & $1,3,4$ & & $B B B B W W W W$ \\
\hline$(1, \overline{2}, \underline{3}, \underline{4}, \overline{5}, \underline{6}, \underline{7}, \underline{8})$ & $1,2,5$ & & $B B W W W B B W$ \\
\hline$(1,2, \overline{3}, \underline{4}, \underline{5}, \overline{6}, \underline{7}, \underline{8})$ & $1,2,3$ & & $B B W W W B B W$ \\
\hline$(\underline{1}, \overline{2}, \underline{3}, \underline{4}, \underline{5}, \underline{6}, \underline{7}, \overline{8})$ & 2,6 & $1,5,7$ & $B B W W B W W B$ \\
\hline$(\underline{1}, 2, \underline{3}, \underline{4}, \underline{5}, \underline{6}, 7, \underline{8})$ & $2,6,7$ & 1 & $B B W W B W W B$ \\
\hline$(\underline{1}, 2, \underline{3}, \underline{4}, \overline{5}, \underline{6}, \underline{7}, \underline{8})$ & $2,5,8$ & 1 & $B B W W W B B W$ \\
\hline$(\underline{1}, \overline{2}, \underline{3}, \underline{4}, \underline{6}, \underline{7}, \overline{8})$ & 2,4 & 1,7 & $B B W W B W W B$ \\
\hline$(\underline{1}, \overline{2}, \overline{3}, \overline{4}, \underline{\overline{5}}, \underline{\overline{6}}, \overline{\overline{7}}, \underline{8})$ & $2,4,7$ & 1,7 & $B B W B W W W B$ \\
\hline$(\underline{1}, 2, \underline{3}, \underline{4}, \overline{5}, \underline{6}, 7, \overline{8})$ & $2,5,7,8$ & 1 & $B B B B W W W W$ \\
\hline$(\underline{1}, 2, \underline{3}, 4,5, \underline{6}, \underline{7}, 8)$ & $2,4,5$ & 1 & $B B B B W W W W$ \\
\hline$(\underline{1}, \overline{2}, \overline{3}, \underline{4}, \underline{5}, \overline{6}, \underline{7}, \underline{8})$ & 2,3 & 1 & $B W W B B B W W$ \\
\hline$(\underline{1}, 2,3, \underline{4}, \underline{5}, \underline{\underline{x}}, \underline{7}, \overline{8})$ & $2,3,6$ & 1,7 & $B W W W B B W B$ \\
\hline$(\underline{1}, \overline{2}, \overline{3}, \underline{\overline{4}}, \underline{\overline{5}}, \underline{6}, \overline{7}, \underline{8})$ & $2,3,6,7$ & 1 & $B W W B B W W B$ \\
\hline$(\underline{1}, 2,3, \underline{4}, \underline{5}, \overline{6}, 7, \underline{8})$ & $2,3,7$ & 1 & $B B W W B B W W$ \\
\hline$(\underline{1}, \underline{2}, 3, \underline{4}, \underline{5}, \underline{6}, \underline{7}, \overline{8})$ & 3,6 & $1,2,7$ & $B B W W W B B W$ \\
\hline$(\underline{1}, \underline{2}, 3, \underline{4}, \underline{5}, \underline{6}, \overline{7}, \underline{8})$ & $3,6,7$ & 1,2 & $B W W B B W W B$ \\
\hline$(\underline{1}, \underline{2}, 3, \underline{4}, \underline{5}, \underline{6}, 7, \overline{8})$ & $3,6,7,8$ & 1,2 & $B W W W B B W B$ \\
\hline$(\underline{1}, \underline{2}, \overline{3}, \underline{4}, \overline{5}, \overline{6}, \underline{7}, \underline{8})$ & 3,5 & 1,2 & $B W W B B B W W$ \\
\hline$(\underline{1}, \underline{2}, 3, \underline{4}, 5, \underline{6}, \underline{7}, \overline{8})$ & $3,5,6$ & 1,2 & $B B B W B W W W$ \\
\hline$(\underline{1}, \underline{2}, \underline{3}, \underline{4}, \underline{5}, \overline{6}, \underline{7}, \underline{8})$ & 4 & $1,2,3,5$ & $B W B W B B W W$ \\
\hline$(\underline{1}, \underline{2}, \underline{3}, \underline{5}, \underline{6}, \underline{7}, 8)$ & 4,5 & $1,2,3$ & $B W W B W W B B$ \\
\hline$(\underline{1}, \underline{2}, \underline{3}, \underline{4}, \overline{5}, \underline{6}, \underline{7}, \underline{8})$ & $4,5,8$ & $1,2,3$ & $B W B W W B B W$ \\
\hline
\end{tabular}

Table B 


\section{Appendix $\mathrm{C}$}

\begin{tabular}{|c|c|c|c|c|c|}
\hline Player & History & InitialConfiguration & Action & BestResponse & FinalConfiguration \\
\hline 1 & 0 & $B W B W B W B W$ & 1 & $(2,3, \overline{4}, 5,6,7, \overline{8})$ & $B B W W B B W W$ \\
\hline 1 & 0 & $B W B W B W B W$ & $\overline{1}$ & $(\underline{2}, \underline{3}, \underline{4}, \overline{\overline{5}}, \underline{6}, \underline{\overline{7}}, \underline{8})$ & $B B W W B B W W$ \\
\hline 2 & 1 & $B W B W B W B W$ & 2 & $(\underline{3}, 4, \underline{5}, \underline{6}, \underline{7}, 8)$ & $B B W W B B W W$ \\
\hline 2 & $\overline{1}$ & $B W B W B W B W$ & $\overline{\overline{2}}$ & $(\underline{3}, \underline{4}, \underline{\overline{6}}, \underline{\overline{7}}, \underline{8}))$ & $B B W W B B W W$ \\
\hline 3 & $(\underline{1}, \underline{2})$ & $B W B W B W B W$ & $\underline{3}$ & $(\overline{4}, 5, \underline{6}, 7, \overline{8})$ & $B B W W B B W W$ \\
\hline 3 & $(\underline{1}, \overline{2})$ & $B W B W B W B W$ & $\overline{\overline{3}}$ & $(\underline{4}, \overline{5}, \overline{6}, \underline{7}, \underline{8}))$ & $B W W B B B W W$ \\
\hline 4 & $(1, \underline{2}, \underline{3})$ & $W B B W B W B W$ & $\underline{4}$ & $(5, \underline{6}, \underline{7}, \underline{8}))$ & $W B B W W B B W$ \\
\hline $4 *$ & $(\overline{1}, \underline{2}, \underline{3})$ & $W B B W B W B W$ & $\overline{\overline{4}}$ & $(\underline{5}, \underline{6}, \overline{7}, \underline{8})$ & $B B B B W W W W$ \\
\hline 5 & $(\underline{1}, 2, \underline{3}, \underline{4})$ & $B B W W B W B W$ & $\underline{5}$ & $(\overline{6}, \underline{7}, \underline{8})$ & $B B W W B B W W$ \\
\hline $5 *$ & $(\underline{1}, \overline{2}, \overline{3}, \underline{4})$ & $B B W W B W B W$ & $\overline{\overline{5}}$ & $(\underline{6}, \overline{\overline{7}}, \overline{\overline{8}})$ & $B B W W W W B B$ \\
\hline 5 & $(\overline{\overline{1}}, \overline{2}, \overline{3}, \underline{4})$ & $B B W W B W B W$ & $\underline{5}$ & $(\overline{\overline{6}}, \overline{\overline{7}}, \underline{8})$ & $B B W W B B W W$ \\
\hline $5 *$ & $(\overline{1}, \overline{2}, \underline{3}, \underline{4})$ & $B B W W B W B W$ & $\overline{\overline{5}}$ & $(\underline{6}, \overline{7}, \overline{8})$ & $B B W W W W B B$ \\
\hline 5 & $(1,2,3,4)$ & $B W B W B W B W$ & 5 & $(\overline{\overline{6}}, \overline{7}, 8)$ & $B W B W B B W W$ \\
\hline 5 & $(1,2,3,4)$ & $B W B W B W B W$ & $\overline{\overline{5}}$ & $(6, \overline{7}, \overline{8})$ & $B W W B W W B B$ \\
\hline 6 & $(1, \underline{2}, \underline{3}, \underline{4}, \underline{5})$ & $W B B W B W B W$ & $\underline{6}$ & $(7, \underline{8})$ & $W B B B W B W W$ \\
\hline 6 & $(\overline{1}, \underline{2}, \underline{3}, \underline{4}, \underline{5})$ & $W B B W B W B W$ & $\overline{\overline{6}}$ & $(\underline{7}, \underline{8})$ & $W B B W B B W W$ \\
\hline 6 & $(\overline{1}, \underline{2}, \overline{3}, \underline{4}, \underline{5})$ & $W B W B B W B W$ & $\underline{6}$ & $(\overline{\overline{7}}, \underline{8})$ & $W B B W B B W W$ \\
\hline $6 *$ & $(\overline{1}, \overline{2}, \overline{3}, 4,5)$ & $W B W B B W B W$ & $\overline{\overline{6}}$ & $(7,8)$ & $W B W B B B W W$ \\
\hline 7 & $(1,2,3,4,5,6)$ & $B B W W B W B W$ & 7 & $\frac{1}{8}=$ & $B B W W W B W B$ \\
\hline 7 & $(\underline{\overline{1}}, \overline{2}, \overline{3}, \underline{4}, \underline{\overline{5}}, \underline{\overline{6}})$ & $B B W W B W B W$ & $\overline{\overline{\bar{V}}}$ & $\underline{8}$ & $B B W W B W W B$ \\
\hline 7 & $(\underline{1}, \overline{2}, \underline{3}, \overline{\overline{4}}, \underline{5}, \underline{6})$ & $B B W B W W B W$ & 7 & $\overline{\overline{8}}$ & $B B W W B W W B$ \\
\hline 7 & $(\underline{1}, \overline{2}, \underline{3}, \overline{4}, \underline{5}, \underline{6})$ & $B B W B W W B W$ & $\overline{\overline{7}}$ & $\underline{8}$ & $B B W B W W W B$ \\
\hline 7 & $(1,2,3,4,5,6)$ & $B W B W B W B W$ & 7 & $\overline{\overline{8}}$ & $B W W B W B W B$ \\
\hline 7 & $\left(1,2,3,4, \frac{1}{5}, 6\right)$ & $B W B W B W B W$ & $\overline{\overline{7}}$ & 8 & $B W B W B W W B$ \\
\hline 7 & $(1,2, \overline{3}, 4,5,6)$ & $B W W B B W B W$ & 7 & $\overline{\overline{8}}$ & $B W W B W B W B$ \\
\hline 7 & $\left(1,2, \overline{3}, 4,5, \frac{1}{5}\right)$ & $B W W B B W B W$ & $\overline{\overline{7}}$ & 8 & $B W B W B W W B$ \\
\hline 7 & $\left(1,2,3, \overline{4}, 5, \frac{1}{6}\right)$ & $B W B B W W B W$ & 7 & $\overline{\overline{8}}$ & $B W W B B W W B$ \\
\hline 7 & $\left(1,2, \frac{1}{3}, \overline{4}, \frac{1}{5}, \frac{1}{6}\right)$ & $B W B B W W B W$ & $\overline{\overline{7}}$ & 8 & $B W B B W W W B$ \\
\hline
\end{tabular}

Table C 